\title{
Calcium response in single osteocytes to locally applied mechanical stimulus: Differences in cell process and cell body
}

\section{AUTHOR(S):}

Adachi, Taiji; Aonuma, Yuki; Tanaka, Mototsugu; Hojo, Masaki; Takano-Yamamoto, Teruko; Kamioka, Hiroshi

\section{CITATION:}

Adachi, Taiji ... [et al]. Calcium response in single osteo cytes to locally applied mechanical stimulus: Differences in cell process and cell body. Journal of Biomechanics 2009, 42(12): 1989-1995

\section{ISSUE DATE:}

2009-08

URL:

http://hdl.handle.net/2433/85228

\section{RIGHT:}

c 2009 Elsevier Ltd. All rights reserved.; This is not the published version. Please cite only the published version.; この論文は出版社版でありませ ん。引用の際には出版社版をご確認ご利用ください。 


\section{Calcium Response in Single Osteocytes to Locally Applied Mechanical Stimulus: Differences in Cell Process and Cell Body}

Taiji Adachi ${ }^{1,2}$, Yuki Aonuma ${ }^{1}$, Mototsugu Tanaka ${ }^{3}$, Masaki Hojo ${ }^{1}$, Teruko Takano-Yamamoto ${ }^{4}$, and Hiroshi Kamioka ${ }^{5}$

${ }^{1}$ Department of Mechanical Engineering and Science, Graduate School of Engineering, Kyoto University, Sakyo, Kyoto 606-8501, Japan

${ }^{2}$ Computational Cell Biomechanics Team, VCAD System Research Program, RIKEN, Hirosawa, Wako 351-0198, Japan

${ }^{3}$ Department of Aeronautics, College of Engineering, Kanazawa Institute of Technology, Ougigaoka, Nonoichi, Ishikawa 921-8501, Japan

${ }^{4}$ Division of Orthodontics and Dentofacial Orthopedics, Graduate School of Dentistry, Tohoku University, Aoba, Sendai 980-8575, Japan

${ }^{5}$ Department of Orthodontics and Dentofacial Orthopedics, Graduate School of Medicine, Dentistry and Pharmaceutical Sciences, Okayama University, Okayama 700-8525, Japan

\section{Corresponding Author:}

Taiji Adachi, Ph.D.

\section{Mailing Address:}

Department of Mechanical Engineering and Science Graduate School of Engineering Kyoto University Yoshida-honmachi, Sakyo, Kyoto 606-8501, Japan Tel\&Fax: +81(75)753-5216

E-Mail: adachi@me.kyoto-u.ac.jp

Number of Words: 2999 words, 3196 words (Revised)

Submitted to the Journal of Biomechanics on January 21th, 2009

Revised on March 29th, 2009 


\section{Abstract}

It is proposed that osteocytes embedded in the bone matrix have the ability to sense deformation and/or damage to the matrix and to feed these mechanical signals back to the adaptive bone remodeling process. When osteoblasts differentiate into osteocytes during the bone formation process, they change their morphology to a stellate form with many slender processes. This characteristic cell shape may underlie the differences in mechanosensitivity between the cell processes and cell body. To elucidate the mechanism of cellular response to mechanical stimulus in osteocytes, we investigated the site-dependent response to quantitatively controlled local mechanical stimulus in single osteocytes isolated from chick embryos, using the technique of calcium imaging. A mechanical stimulus was applied to a single osteocyte using a glass microneedle targeting a microparticle adhered to the cell membrane by modification with a monoclonal antibody OB7.3. Application of the local deformation induced calcium transients in the vicinity of the stimulated point and caused diffusive wave propagation of the calcium transient to the entire intracellular region. The rate of cell response to the stimulus was higher when applied to the cell processes than when applied to the cell body. In addition, a large deformation was necessary at the cell body to induce calcium transients, whereas a relatively small deformation was sufficient at the cell processes, suggesting that the mechanosensitivity of the cell processes was higher than that of the cell body. These results suggest that the cell shape with slender processes contributes to the site-dependent mechanosensitivity in osteocytes.

Keywords: Osteocytes, Bone cells, Calcium signaling response, Mechanical stimulus, Mechanotransduction, Cell biomechanics 


\section{Introduction}

Osteocytes are differentiated from osteoblasts, and become enveloped during the bone formation process in adaptive remodeling (Parfitt. 1994). In this process, they change their morphology into a stellate form that consists of a cell body with a nucleus and many slender processes, constructing intercellular networks with surrounding cells within the bone matrix as well as on the bone surface (Kamioka, et al. 2001). This broad bone-cell-network is believed to play a significant role in the functional adaptation to mechanical loading of the bone. In this network, the osteocytes are thought to be the major cells responsible for sensing mechanical stimulus (stress/strain) and sending out signals that coordinate the adaptive remodeling response (Burger and Klein-Nulend. 1999).

Although the character of the mechanical signal that acts on osteocytes as a result of bone loading is still unknown, several potential stimuli affecting osteocyte function are proposed, such as hydrostatic pressure, fluid flow induced shear stress, bone tissue strain, and localized mechanical stimulation by poking (Burger and Klein-Nulend. 1999; Klein-Nulend, et al. 1995; Miyauchi, et al. 2000; Vatsa, et al. 2006; Weinbaum, et al. 1994). In the bone matrix, the mechanical stimulus sensed by the osteocytes could be affected by the complicated mechanical environment associated with their characteristic morphology and internal structures. For example, focal adhesion complexes, connecting intracellular actin filaments with transmembrane integrin receptors, are localized at the proximal base of the osteocytic processes (Kamioka, et al. 2006). This localization may cause concentration of deformation/strain of the osteocytic membrane (Wang, et al. 2007), when the drag force acting on the pericellular matrix caused by fluid flow deforms the cell processes. Therefore, it is possible that osteocytes have the potential to respond to direct deformation of their processes at microscopic levels, however, verification of the site-dependency of the response has not been performed. To examine the importance of the osteocyte processes in the response 
to mechanical stimuli, it is critical to compare the response that arises from the processes and from the cell body in single isolated osteocytes.

One of the earliest events in mechanotransduction is intracellular calcium signaling, which acts as a messenger to activate various cellular functions (Ajubi, et al. 1999; Berridge, et al. 1998; Chen, et al. 2000; You, et al. 2001). In addition, calcium transients in osteocytes, induced by local deformation of the cell membrane, are propagated to the neighboring cells (Gu, et al. 2007; Yellowley, et al. 2000), suggesting this as a mechanism of communication and synchronization in the intercellular network. Therefore, measurement of mechanically induced intracellular calcium transients is thought to be a useful indicator for evaluating the short-term mechanosensory response in bone cells.

In this study, we examined site-dependent calcium transients in single primary osteocytes. In particular, calcium transients in response to direct deformation of osteocytic processes and of the cell body were examined, in which chick-osteocyte-specific-antibody (OB7.3) coated microparticles adhered to the osteocyte surface were employed as a marker for identification of the osteocytes and as a target for the quantification of the deformation applied to the osteocytes.

\section{Materials and Methods}

\section{Isolation and culture of osteocytes}

Osteocytes were isolated from 13-day-old embryonic chicken calvariae with modification of the method described in Kamioka et al. (2004). To release bone cells from the bone matrix, the calvariae were cut into small pieces and shake-incubated, with $1 \mathrm{mg} / \mathrm{ml}$ collagenase type I, in a bone isolation buffer (Hefley. 1987) for $30 \mathrm{~min}$ at $37.5^{\circ} \mathrm{C}$. To decalcify the bone matrix and to expose collagen fibers with bone cells, the calvariae were incubated twice with $0.5 \mathrm{mM}$ EDTA in DPBS containing 0.1\% BSA for 15 min each. To enzymatically 
digest collagen and to release bone cells from the collagen fibers, the decalcified matrix was treated again with the collagenase for $30 \mathrm{~min}$ at $37.5^{\circ} \mathrm{C}$.

The solution was centrifuged for $5 \mathrm{~min}$ at $1200 \mathrm{rpm}$ and the pellet was dispersed in $\alpha$-MEM with 2\% FBS and antibiotics. To separate osteocytes from mixed bone cells, the suspension was filtered using a membrane filter (Millipore) with a pore size of $\phi=10.0 \mu \mathrm{m}$, and then the medium containing osteocytes were collected. The medium was centrifuged and the pellet was dispersed in a 1:1 mixture of $0.5 \% \mathrm{NaCl}$ and a fresh medium to get rid of the cells other than osteocytes. The mixture was diluted with a fresh medium and centrifuged again, and the osteocytes were collected. Then, the isolated cells were seeded onto $\phi=35 \mathrm{~mm}$ glass bottom dishes (MatTek), coated with $20 \mu \mathrm{g} / \mathrm{ml}$ poly-D-lysine (Sigma) and $20 \mu \mathrm{g} / \mathrm{ml}$ fibronectin (human plasma, Chemicon Int.), at a cell density of $7.5 \times 10^{3}$ to $2.0 \times 10^{4} \mathrm{cell} / \mathrm{dish}$. After incubation for $1 \mathrm{~h}$, the cells were rinsed with DPBS and incubated again for 15-18 $\mathrm{h}$ in a fresh medium at $37^{\circ} \mathrm{C}, 100 \%$ relative humidity, and $5 \% \mathrm{CO}_{2}$ in air.

\section{Surface treatment of microparticles with antibody for attachment to cell membrane}

Polystyrene microparticles ( $\phi=1.0 \mu \mathrm{m}$, Polysciences) were suspended in $20 \mu \mathrm{g} / \mathrm{ml}$ polyethylene glycol for 60 min at $37^{\circ} \mathrm{C}$, collected by centrifugation, and then suspended in a medium with monoclonal antibody OB7.3 (a kind gift from Jenneke Klein-Nulend and Cornelis M. Semeins, ACTA-Vrije University) (diluted to 1:5 in $\alpha$-MEM) for $30 \mathrm{~min}$. The microparticles with the immersion coating of OB7.3 adhered selectively to the cell membrane of osteocytes. The OB7.3-coated microparticles were then suspended in a medium with calcium fluorescence indicators, as described below, and were incubated for $15 \mathrm{~min}$ at $37^{\circ} \mathrm{C}$ to allow the microparticles to adhere to the cell membrane.

To confirm microparticle coating with OB7.3 and adhesion of the coated microparticles to the osteocytes, we observed the microparticles with immunofluorescence 
labeling of with Alexa Fluor 546 goat anti-mouse IgG. In addition, attachment of the microparticles was verified based on field emission scanning electron microscopy (S-4700, Hitachi) observations.

\section{Application of localized deformation}

A localized mechanical stimulus was applied to a single osteocyte using a glass microneedle via microparticles attached on the cell membrane, as illustrated in Fig.1. A microneedle, with a tip diameter of about $\phi=2 \mu \mathrm{m}$, was attached to a hydraulic micromanipulator (MM-188NE, Narishige), and set up on the microscope stage at an angle of 40 degrees from the dish plane. To quantitatively apply the mechanical stimulus to the cell, the microneedle displacement was controlled using a custom-made apparatus, as described by Sato et al. (2007), with a linear slider and a piezoelectric actuator (Physik Instrumente) at a constant velocity $v=0.5 \mu \mathrm{m} / \mathrm{s}$.

Using phase-contrast microscopy, position was traced and displacement of the microparticles $\delta(t)$ was determined. A series of images was obtained at $0.25 \mathrm{~s}$ intervals, and detection of the initial movement of the targeted microparticle was defined as $t=0 \mathrm{~s}$ for the measurement.

\section{Calcium imaging}

The concentration of intracellular calcium ions, $\left[\mathrm{Ca}^{2+}\right]_{i}$, was evaluated using a ratiometric method (Grynkiewicz, et al. 1985). Two fluorescent calcium indicators, $6 \mu \mathrm{M}$ Fluo-4 AM and Fura Red AM (Invitrogen), were loaded into the cells with a solubilizing agent, $0.01 \%$ Cremophor EL (Sigma-Aldrich), for $15 \mathrm{~min}$ at $37^{\circ} \mathrm{C}$. Subsequently, the cells were rinsed with DPBS, and the medium was replaced with a $\mathrm{CO}_{2}$ independent medium for pH stabilization.

The calcium response was observed using a confocal laser-scanning microscope (LSM 
510, Carl Zeiss). Both calcium indicators were excited at $488 \mathrm{~nm}$ wavelength simultaneously, and a band pass filter (green: 505-530 nm) and a long pass filter (red: > $560 \mathrm{~nm}$ ) were used to detect the fluorescence of Fluo-4 (516 nm peak) and Fura Red (599 nm peak), respectively.

To define a responsive cell, we adopted a criteria based on a previous study (Hung, et al. 1995), that is, cells with a transient increase in $\left[\mathrm{Ca}^{2+}\right]_{i}$ of 1.5 times the baseline value were classified as responsive cells. We measured the percentage of cells that were responsive to mechanical stimulus applied to the cell body and to cell processes, and compared them by performing a Fisher's exact probability test with a statistical significant level of $p<0.01$. In addition, we measured the magnitude of mechanical stimulus $\delta_{\text {res }}$ that induced the calcium transient, and compared for the cell body and cell processes by performing a student's t-test with a statistical significant level of $p<0.05$.

\section{Results}

\section{Identification of cell phenotype and adhesion of microparticles}

The OB7.3-coated microparticles, via which mechanical stimuli were applied to the cell, were also used as a marker of osteocytes. Microparticles, dispersed at a density of $1.8 \times$ $10^{9}$ particles $/ \mathrm{cm}^{3}$ and washed by DPBS, were found to adhere stably to the cell membrane even after washing (Fig.2A). Fluorescent immunolabeling (Fig.2B) of the same cell (Fig.2A) confirmed that all observed microparticles were coated with OB7.3.

Scanning electron microscopic images showed the attachment sites of the microparticles to the osteocytes (Fig.2C). The OB7.3-coated microparticles were observed in both the cell processes and body, and stably attached to the cell membrane as shown in the magnified images in Fig.2C.

\section{Calcium response to locally applied mechanical stimulus}

Changes in $\left[\mathrm{Ca}^{2+}\right]_{i}$ in an osteocyte were observed in response to local deformation via 
a microparticle moved by a microneedle for the cell process (Figs.3A-D) and cell body

(Figs.3E-H). Microparticles targeted for deformation are shown in superimposed images of the transmitted and fluorescent images (Figs.3A and E, white arrow). Pseudo-colored ratio images at $t=0 \mathrm{~s}$ are shown in Figs.3B and F, and their time-lapse images in Figs.3C and G. When the targeted microparticle adhered on the osteocyte process displaced by the microneedle (white arrowhead in Fig.3B), a rapid calcium transient was observed in the region adjacent to the microparticle. In addition, the calcium transient propagated to the whole cell body and even to other cell processes (Fig.3C). Contrary to this, a relatively slow response was observed for the cell body (Fig.3G).

To evaluate the intracellular propagation of the calcium transient, the changes in $\left[\mathrm{Ca}^{2+}\right]_{i}$ from two different rectangular regions, I and II (Figs.3A and E), were examined (Figs.3D and H). For the cell process, in the region proximal to the deformed process (I), the calcium transient increased rapidly from a baseline at $t=3.75 \mathrm{~s}$, reached maximum at $t=5.75$ $\mathrm{s}$ and then declined gradually. In the region distal to the deformed process (II), $10 \mu \mathrm{m}$ from region I, the calcium transient was seen after an additional $0.25 \mathrm{~s}$ interval. These results indicate that the calcium transient starts from the deformed site and propagates to the whole cell body within one second. In comparison with this, for the cell body, the calcium transient seemed to propagate (Fig.3G), but it was hard to clearly demonstrate it using the temporal resolution of $0.25 \mathrm{~s}$, because of the relatively slow increase in $\left[\mathrm{Ca}^{2+}\right]_{i}($ Fig.3H). Despite this difference in the response, the peak calcium amplitude was comparable in either the cell process or the cell body (data not shown).

Cells were divided into two groups according to the targeted position of the applied stimulus, the cell body or the cell process, and the percentage of cells responding to the stimulus in each position was determined (Fig.4). It was found that the percentage of cells responding to the applied stimulus was higher in the cell process (40.0\%) than in the cell body 
(8.5\%) with a significant difference $(p<0.01)$ by a Fisher’s exact probability test.

The amount of mechanical stimulus that caused a calcium transient in response to the local deformation of the cell body and cell process is represented as the microparticle displacement $\delta_{\text {res }}$, and is shown in Fig.5. Interestingly, the amount of mechanical stimulus needed to elicit a calcium transient was different in the cell body ( $4.3 \pm 0.8 \mu \mathrm{m})$ and in the cell process. $(2.0 \pm 0.5 \mu \mathrm{m})$, and showed a statistically significant difference $(p<0.05)$ by t-test. These results show that a smaller deformation was enough to elicit a calcium transient at the cell processes compared to that required at the cell body, implying that sensitivity to mechanical stimulus is higher in the cell processes.

\section{Discussion}

Osteocytes in the bone matrix are located in cavities called lacunae, and they elongate their processes into canaliculi. Therefore, the cell processes and cell body of the osteocyte are thought to exist in different mechanical microenvironments, that is, the mechanical stimuli are dependent on each specific mechanical microenvironment. However, the site-specific mechanical responses in the osteocyte have not been examined.

We previously reported that there are differences in the actin cytoskeletal structure between the cell processes and cell body, and we showed that the osteocyte processes are rich in actin filaments with higher elastic modulus than the cell body (Sugawara, et al. 2008; Tanaka-Kamioka, et al. 1998). These unique internal structures and the site-dependence of the elastic modulus could modulate the sensitivity to direct deformation (Adachi, et al. 2003; Wu, et al. 1999). Therefore, in this study, we developed an experimental method to apply a quantitative local mechanical stimulus to a single osteocyte and compared site-specific calcium responses between the cell processes and cell body. 


\section{Application of local mechanical stimulus in single primary osteocyte}

In this study, to apply a quantitative local mechanical stimulus to a single osteocyte, we developed an experimental system using a microneedle and antibody-coated microparticles. A monoclonal antibody, OB7.3, that specifically recognizes transmembrane Phex protein of chicken osteocytes (Rowe. 1997; Westbroek, et al. 2002), has been reported to be useful for the panning method of osteocyte isolation due to its strong binding to the cell membrane (van der Plas and Nijweide. 1992). Therefore, the OB7.3-coated microparticle binding enabled us to deform osteocyte membranes indirectly using a microneedle, and to quantify the local deformation by measuring the displacement of the microparticle. We have found that the similar calcium transient was observed when the cell membrane was deformed directly using a tip of the microneedle. However, the tip does not stably stick on the cell membrane and often slips relative to the membrane (Adachi, et al. 2008; Sato, et al. 2007). Therefore, we utilized microparticles directly adhered to the membrane, by which we could have advantage to quantitatively evaluated the deformation.

Similar stimulation was performed using a microparticle coated by fibronectin, a known signal transducing transmembrane molecule. The fibronectin-coated microparticles adhered to the osteocytic membrane; however, we rarely observed microparticles spread over the cell surface from the processes to the cell body. This inhomogeneous adhesion indicates localized distribution of the focal adhesions or different adhesion characteristics for the cell processes and cell body. Actually, some microparticles adhere to the peripheral region of the cell body and cell processes, but not many on the apical surface of the cell body. Therefore, to identify differences in mechanosensitivity of the cell processes and cell body, we needed to utilize a technique by which we could attach microparticles on both the cell processes and cell body. Monoclonal antibody OB7.3 is known to cover the entire surface of osteocytes (Kamioka, et al. 2006), therefore, it is an appropriate tool for our purpose. 
We defined the measure of the mechanical stimulus to the osteocytes as the microparticle displacement, because we assume that the essential mechanical stimulus is the relative perturbation to the cell membrane with respect to a fixed-point support such as focal adhesions, as discussed by Bonewald (2006) and Wang et al. (2007). The experimental system in this study has been designed based on the hypothesized mechanism as illustrated in Fig.6. That is, the mechanical stimulus to the cell processes and cell body is applied to a local point and the transmitted force focuses on focal adhesions, to which the mechanosensitive (MS) channel is believed to interferes or connects (Hayakawa, et al. 2008), via the internal structures such as the cytoskeletal actin structure.

In this experimental system, the displacement of the microparticle was quantitatively applied under piezoelectric control; however, the force could not be measured. A new in vitro technique that will enable the measurement of the force at pN level (Wang, et al. 2007) is strongly desired in order to better understand the mechanosensitivity in osteocytes. Nonetheless, the insight into the difference in response to the mechanical stimulus between the cell processes and cell body provides useful and important information as a first step for studying not only mechanosensing mechanisms but also the biological implications of the stellate cell shape with its slender processes.

\section{Mechanosensitivity in cell processes and cell body}

Adhesive structures that transmit the microparticle displacement to the internal osteocytes in this in vitro study correspond to the adhesive structures in vivo to the canalicular wall. Indeed, it was reported that the osteocytes attach to the wall in canaliculi mediated by tethering elements (Wang, et al. 2007; You, et al. 2004), and that the focal adhesions are distributed at the proximal base of the cell processes and cell body in the bone matrix (Kamioka, et al. 2006; Vatsa, et al. 2008). A schematic of the hypothesized mechanosensing mechanism via the adhesive structure is illustrated in Fig.6A. When the axial interstitial fluid 
flow in the canaliculi drags cell processes via tethering elements, the relative displacement with respect to the locally fixed adhesive structures generates local reaction forces at the canalicular projection, which is transmitted via mediated molecules and actin cytoskeleton, and result in the activation of MS channels in the vicinity of the focal adhesions (Han, et al. 2004; Wang, et al. 2007; You, et al. 2001).

Similarly, mechanical stimulus-induced calcium transient in this study could be understood by the mechanism illustrated in Fig.6B, in which the microparticles correspond to the tethering element as depicted in Fig.6A. If a similar mechanical signal transmission to a fixed-point support such as integrin through the intracellular structure, the observed calcium transient could be understood through the same mechanism, implying that the cell processes equip the mechanosensitivity to the local mechanical stimulus via adhesive and internal structures.

Higher sensitivity to the mechanical stimulus in the cell processes than the cell body might be due to the differential localization of actin filament (Tanaka-Kamioka, et al. 1998), as illustrated in Fig.6C. This implies that the force transmission from the displaced microparticle with concentrated deformation (Adachi, et al. 2008; Sato, et al. 2007) to the fixed point plays a pivotal role. Here, we should emphasizes that this stress/strain concentration might also be involved in the amplification of the mechanosensing mechanism of the osteocytes, which can be an additional factor affecting the strain amplification mechanism (Wang, et al. 2007).

In contrast with fluid shear stress experiments on the osteocytes (Reilly, et al. 2003; You, et al. 2000), the method developed in this study applies mechanical stimulus under displacement-controlled condition. One of the limitations of this study is that we still cannot clearly understand in vivo mechanical conditions of the osteocytes to reflect to the in vitro experiment, such as forces acting on the cell and their corresponding displacements, which 
may greatly differ from the in vitro experimental conditions due to the complex lacuno-canalicular geometry and the distribution of focal adhesions. Therefore, considering the displacement-controlled experiment as one of limit cases for the mechanical conditions, understanding of the response to direct deformation of the osteocytes would be useful to gain further insights into their mechanosensing mechanism.

\section{Conflict of Interest}

None.

\section{Acknowledgement}

Monoclonal antibody OB7.3 was kindly provided by Jenneke Klein-Nulend and Cornelis M. Semeins (ACTA-Vrije University). FE-SEM observations were assisted by Laboratory for Electron Microcopy, Center for Anatomical Studies, Graduate School of Medicine, Kyoto University. This study was partially supported by the Grants-in-Aid for Specially Promoted Research (20001007) from the Ministry of Education, Culture, Sports, Science and Technology, Japan, and by the Japan Society for the Promotion of Science under the Research Fellowships for Young Scientists. 


\section{References}

Adachi, T., Sato, K., Higashi, N., Tomita, Y., and Hojo, M. 2008. Simultaneous observation of calcium signaling response and membrane deformation due to localized mechanical stimulus in single osteoblast-like cells. J Mech Behav Biomed Mater. 1: 43-50.

Adachi, T., Sato, K., and Tomita, Y. 2003. Directional dependence of osteoblastic calcium response to mechanical stimuli. Biomech Model Mechanobiol. 2: 73-82.

Ajubi, N.E., Klein-Nulend, J., Alblas, M.J., Burger, E.H., and Nijweide, P.J. 1999. Signal transduction pathways involved in fluid flow-induced PGE2 production by cultured osteocytes. Am J Physiol. 276: E171-178.

Berridge, M.J., Bootman, M.D., and Lipp, P. 1998. Calcium - a life and death signal. Nature. 395: 645-648.

Bonewald, L.F. 2006. Mechanosensation and transduction in osteocytes. Bonekey Osteovision. 3: 7-15.

Burger, E.H., and Klein-Nulend, J. 1999. Mechanotransduction in bone - role of the lacuno-canalicular network. FASEB J. 13: S101-112.

Chen, N.X., Ryder, K.D., Pavalko, F.M., Turner, C.H., Burr, D.B., Qiu, J., and Duncan, R.L. 2000. Ca2+ regulates fluid shear-induced cytoskeletal reorganization and gene expression in osteoblasts. Am J Physiol Cell Physiol. 278: C989-997.

Grynkiewicz, G., Poenie, M., and Tsien, R.Y. 1985. A new generation of Ca2+ indicators with greatly improved fluorescence properties. J Biol Chem. 260: 3440-3450.

Gu, G., Lindqvist, R., and Väänänen, H.K. 2007. Mechanical stimulation of a single osteocyte induce intercellular calcium waves through the osteocyte network. Calcif Tis Int. 80: S77.

Han, Y., Cowin, S.C., Schaffler, M.B., and Weinbaum, S. 2004. Mechanotransduction and strain amplification in osteocyte cell processes. Proc Natl Acad Sci USA. 101: 16689-16694.

Hayakawa, K., Tatsumi, H., and Sokabe, M. 2008. Actin stress fibers transmit and focus force to activate mechanosensitive channels. J Cell Sci. 121: 496-503.

Hefley, T.J. 1987. Utilization of FPLC-purified bacterial collagenase for the isolation of cells from bone. J Bone Miner Res. 2: 505-516.

Hung, C.T., Pollack, S.R., Reilly, T.M., and Brighton, C.T. 1995. Real-time calcium response of cultured bone cells to fluid flow. Clin Orthop Relat Res. 313: 256-269.

Kamioka, H., Honjo, T., and Takano-Yamamoto, T. 2001. A three-dimensional distribution of osteocyte processes revealed by the combination of confocal laser scanning microscopy and differential interference contrast microscopy. Bone. 28: 145-149.

Kamioka, H., Sugawara, Y., Murshid, S.A., Ishihara, Y., Honjo, T., and Takano-Yamamoto, T. 2006. Fluid shear stress induces less calcium response in a single primary osteocyte than in a single osteoblast: Implication of different focal adhesion formation. $J$ Bone Miner Res. 21: 1012-1021.

Klein-Nulend, J., van der Plas, A., Semeins, C.M., Ajubi, N.E., Frangos, J.A., Nijweide, P.J., and Burger, E.H. 1995. Sensitivity of osteocytes to biomechanical stress in vitro. FASEB J. 9: 441-445.

Miyauchi, A., Notoya, K., Mikuni-Takagaki, Y., Takagi, Y., Goto, M., Miki, Y., Takano-Yamamoto, T., Jinnai, K., Takahashi, K., Kumegawa, M., Chihara, K., and Fujita, T. 2000. Parathyroid hormone-activated volume-sensitive calcium influx pathways in mechanically loaded osteocytes. J Biol Chem. 275: 3335-3342.

Parfitt, A.M. 1994. Osteonal and hemi-osteonal remodeling - the spatial and temporal framework for signal traffic in adult human bone. Journal of Cellular Biochemistry. 
55(3): 273-286.

Reilly, G.C., Haut, T.R., Yellowley, C.E., Donahue, H.J., and Jacobs, C.R. 2003. Fluid flow induced PGE2 release by bone cells is reduced by glycocalyx degradation whereas calcium signals are not. Biorheology. 40: 591-603.

Rowe, P.S. 1997. The PEX gene: its role in X-linked rickets, osteomalacia, and bone mineral metabolism. Exp Nephrol. 5: 355-363.

Sato, K., Adachi, T., Ueda, D., Hojo, M., and Tomita, Y. 2007. Measurement of local strain on cell membrane at initiation point of calcium signaling response to applied mechanical stimulus in osteoblastic cells. J Biomech. 40: 1246-1255.

Sugawara, Y., Ando, R., Kamioka, H., Ishihara, Y., Murshid, S.A., Hashimoto, K., Kataoka, N., Tsujioka, K., Kajiya, F., Yamashiro, T., and Takano-Yamamoto, T. 2008. The alteration of a mechanical property of bone cells during the process of changing from osteoblasts to osteocytes. Bone. 43: 19-24.

Tanaka-Kamioka, K., Kamioka, H., Ris, H., and Lim, S.S. 1998. Osteocyte shape is dependent on actin filaments and osteocyte processes are unique actin-rich projections. $J$ Bone Miner Res. 13: 1555-1568.

van der Plas, A., and Nijweide, P.J. 1992. Isolation and purification of osteocytes. J Bone Miner Res. 7: 389-396.

Vatsa, A., Mizuno, D., Smit, T.H., Schmidt, C.F., MacKintosh, F.C., and Klein-Nulend, J. 2006. Bio imaging of intracellular NO production in single bone cells after mechanical stimulation. J Bone Miner Res. 21: 1722-1728.

Vatsa, A., Semeins, C.M., Smit, T.H., and Klein-Nulend, J. 2008. Paxillin localisation in osteocytes-Is it determined by the direction of loading? Biochemical and Biophysical Research Communications. 377(4): 1019-1024.

Wang, Y., McNamara, L.M., Schaffler, M.B., and Weinbaum, S. 2007. A model for the role of integrins in flow induced mechanotransduction in osteocytes. Proc Natl Acad Sci USA. 104: 15941-15946.

Weinbaum, S., Cowin, S.C., and Zeng, Y. 1994. A model for the excitation of osteocytes by mechanical loading-induced bone fluid shear stresses. J Biomech. 27: 339-360.

Westbroek, I., De Rooij, K.E., and Nijweide, P.J. 2002. Osteocyte-specific monoclonal antibody MAb OB7.3 is directed against Phex protein. J Bone Miner Res. 17: 845-853.

Wu, Z., Wong, K., Glogauer, M., Ellen, R.P., and McCulloch, C.A. 1999. Regulation of stretch-activated intracellular calcium transients by actin filaments. Biochem Biophys Res Commun. 261: 419-425.

Yellowley, C.E., Li, Z., Zhou, Z., Jacobs, C.R., and Donahue, H.J. 2000. Functional gap junctions between osteocytic and osteoblastic cells. J Bone Miner Res. 15: 209-217.

You, J., Reilly, G.C., Zhen, X., Yellowley, C.E., Chen, Q., Donahue, H.J., and Jacobs, C.R. 2001. Osteopontin gene regulation by oscillatory fluid flow via intracellular calcium mobilization and activation of mitogen-activated protein kinase in MC3T3-E1 osteoblasts. J Biol Chem. 276: 13365-13371.

You, J., Yellowley, C.E., Donahue, H.J., Zhang, Y., Chen, Q., and Jacobs, C.R. 2000. Substrate deformation levels associated with routine physical activity are less stimulatory to bone cells relative to loading-induced oscillatory fluid flow. J Biomech Eng. 122: 387-393.

You, L., Cowin, S.C., Schaffler, M.B., and Weinbaum, S. 2001. A model for strain amplification in the actin cytoskeleton of osteocytes due to fluid drag on pericellular matrix. Journal of Biomechanics. 34: 1375-1386.

You, L.D., Weinbaum, S., Cowin, S.C., and Schaffler, M.B. 2004. Ultrastructure of the osteocyte process and its pericellular matrix. Anat Rec A Discov Mol Cell Evol Biol. 278: 505-513. 


\section{Figure Legends}

\section{Figure 1}

Schematic of mechanical stimulus application to an osteocyte. Local mechanical stimulus was applied using a glass microneedle via a microparticle attached to an osteocytic cell membrane whose surface was treated with a monoclonal antibody OB7.3. A linear slider and a piezoelectric actuator were utilized to control the microneedle displacement horizontally and were moved at a constant velocity $(0.5 \mu \mathrm{m} / \mathrm{s})$ as indicated by an arrow. We defined the time $t$ $=0 \mathrm{~s}$ as the initial movement of the targeted microparticle, and the displacement $\delta$ of the microparticle was measured as the amount of the mechanical stimulus applied to the osteocytes.

\section{Figure 2}

An isolated osteocyte with a microparticle attached on the cell membrane. (A) and (B): Surface coating of microparticles with OB7.3 was confirmed using anti-mouse goat IgG antibody labeled with Alexa Fluor 546. All of the microparticles on the cell surface in the transmitted image (A) were labeled with indirect immunofluorescence in image (B). Bar = 10 $\mu \mathrm{m}$. (C): FE-SEM images of an osteocyte with microparticles, taken at a tilted angle of $45^{\circ}$. Two images in the right are magnified views of the left image around attached microparticles. The OB7.3-coated microparticles were attached to both the cell body and cell processes. Bar $=10 \mu \mathrm{m}$ (left) and $2 \mu \mathrm{m}$ (both right).

\section{Figure 3}

Calcium response induced by application of local mechanical stimulus when the stimulus was applied to the cell process (A-D) and the cell body (E-H). (A, E): Superimposed images of transmitted and fluorescent images. The microparticles displaced by the microneedle are 
indicated by white arrows. To measure the intracellular calcium propagation, the fluorescence ratios of two fluorescent indicators $\left(R_{\mathrm{F} 4 / \mathrm{FR}}\right)$ corresponding to $\left[\mathrm{Ca}^{2+}\right]_{i}$, normalized by their initial values, were measured in two different square regions, Regions I and II. Bar $=10 \mu \mathrm{m}$. (B, F): Fluorescence ratio images at $t=0 \mathrm{~s}$ displayed using pseudo-color. Positions of the tip of the microneedle are indicated by arrowheads. (C, G): Time-lapse images from $t=$ $3.00-4.25 \mathrm{~s}$ for the cell process, and those from $t=7.00-8.25 \mathrm{~s}$ for the cell body. Positions of tip of the microneedles at that time are indicated by arrowheads. (D, H): Time courses of change in fluorescence ratio, measured in the Regions I and II displayed in (A, E), were obtained every $0.25 \mathrm{~s}$. For the cell process, a rapid transient increase in the ratio was observed, and the intracellular propagation of the calcium response was clearly observed by the delay in the increase in the ratio in Region II from Region I; whereas a relatively slow increase in the ratio was observed for the cell body.

\section{Figure 4}

Percentage of cells responding to local deformation in the cell populations in which the microparticles were successfully displaced by the microneedle. Cells were divided into two groups by the targeted position of the applied stimulus. The percentage of responsive cells when the cell process was stimulated was higher compared to that when the cell body was stimulated. (Fisher's exact probability test $* *: p<0.01$ )

\section{Figure 5}

Stimulus that induces calcium transient. Microparticle displacement $\delta_{\text {res }}$ required to cause calcium transient was recorded for every responsive cell. Values are expressed as the Mean \pm SE for responsive cells in each group. A smaller deformation was sufficient at the cell processes to induce a calcium response compared to that at the cell body. (t-test $*: p<0.05$ ). 


\section{Figure 6}

Mechanosensing mechanism in osteocytes hypothesized in this study. (A): In canaliculi, osteocyte cell processes are anchored by tethering transverse elements that span the entire pericellular space from the cell membrane to the canalicular wall (You, et al. 2004), and they are presumed to attach directly to canalicular projections via focal adhesion molecules (Wang, et al. 2007). Interstitial fluid flow generated by mechanical loading causes a relative perturbation between the cell process membrane and the canalicular wall, followed by a local mechanical stimulus input via adhesion molecules. The induced mechanical stimulus is transmitted to adhesion molecules such as integrins through the internal structures such as actin filaments, and activates MS channels in the vicinity of integrin, which results in calcium transient. (B): A mechanism of calcium transient induced in our in vitro experiment. Displacement of an OB7.3 coated microparticle applies a local mechanical stimulus input to Phex protein on the cell surface. The mechanical stimulus is transmitted and focused to the focal attachment via intracellular structures such as the actin filament. (C): Application of a local mechanical stimulus to the osteocyte cell process and cell body in vitro. Difference in distance between stimulated and fixed points and in their stiffness could contribute to the different mechanosensitivity. 
Figure 1

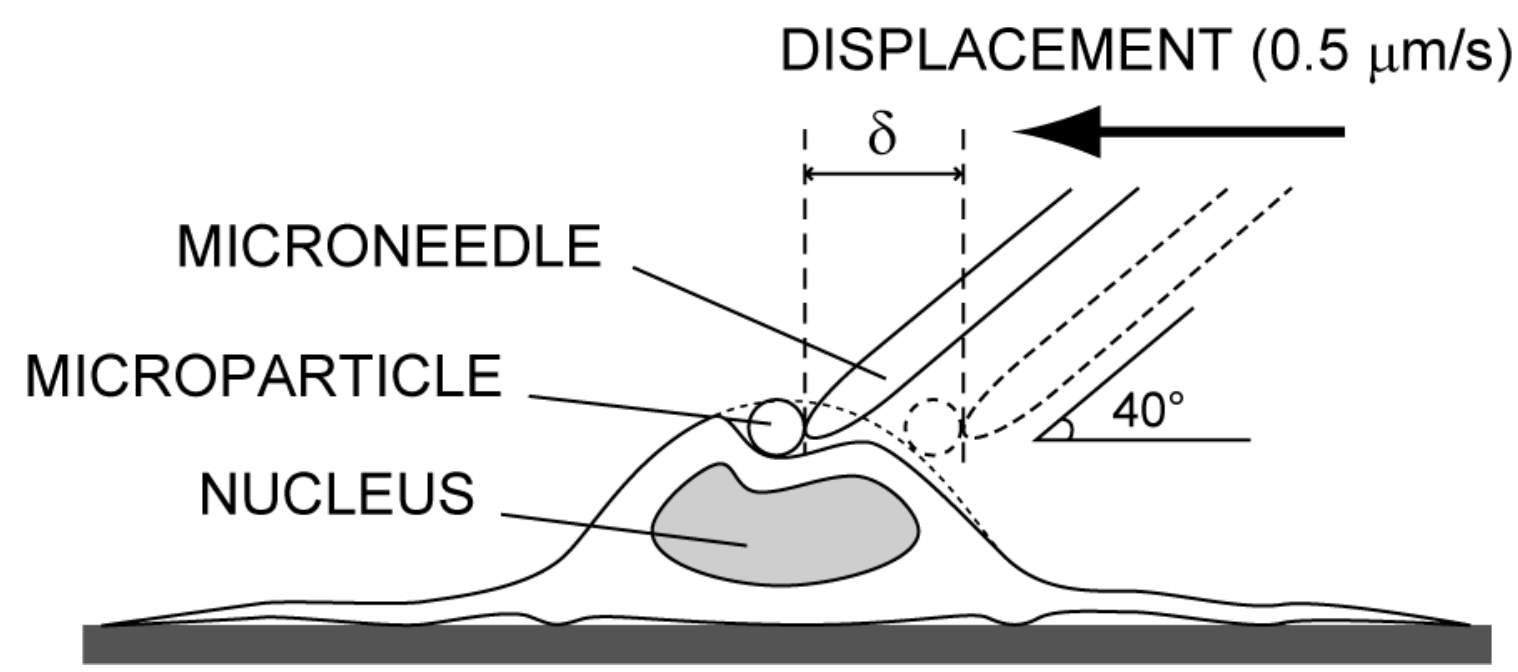


Figure 2

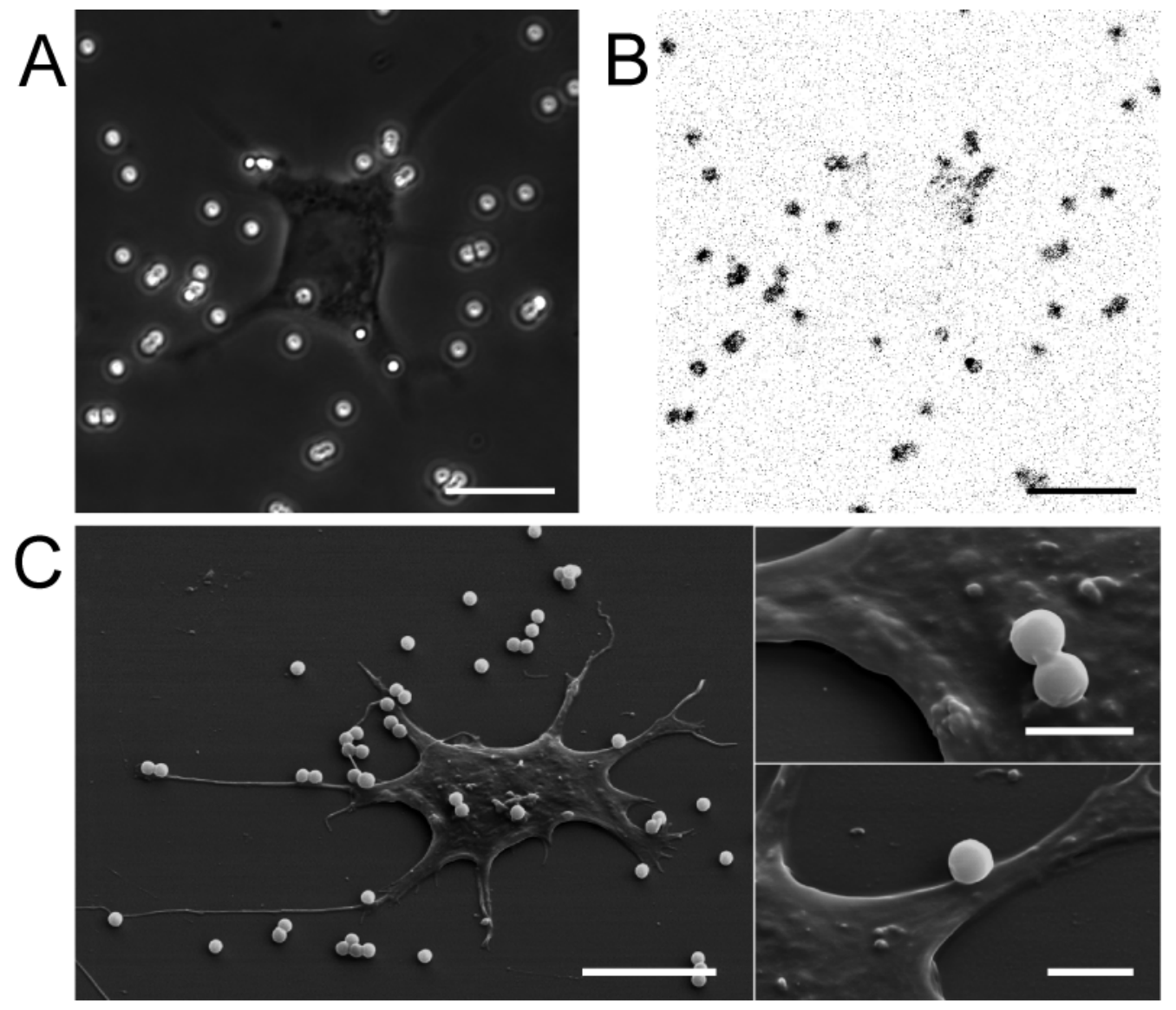


Figure 3
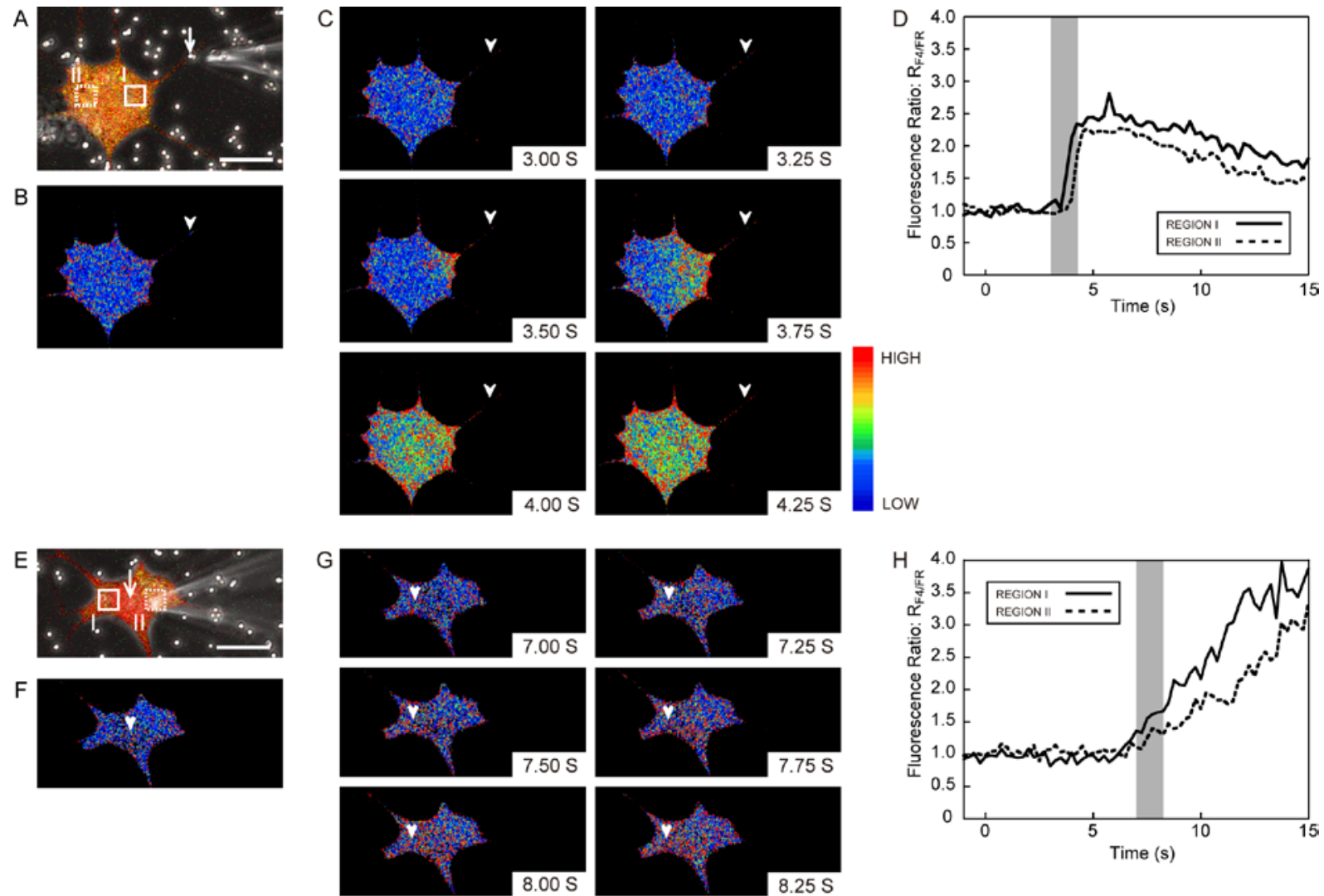
Figure 4

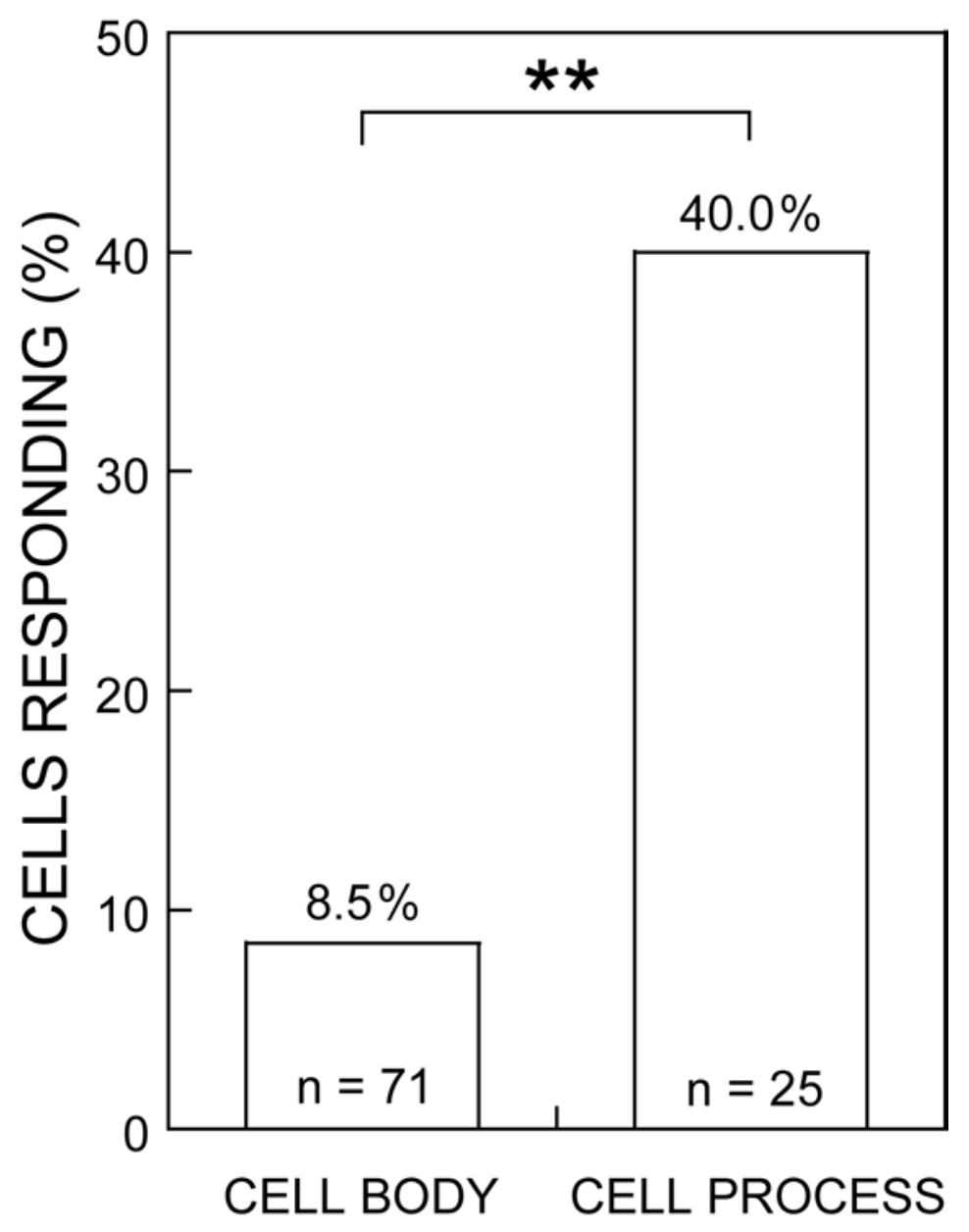


Figure 5

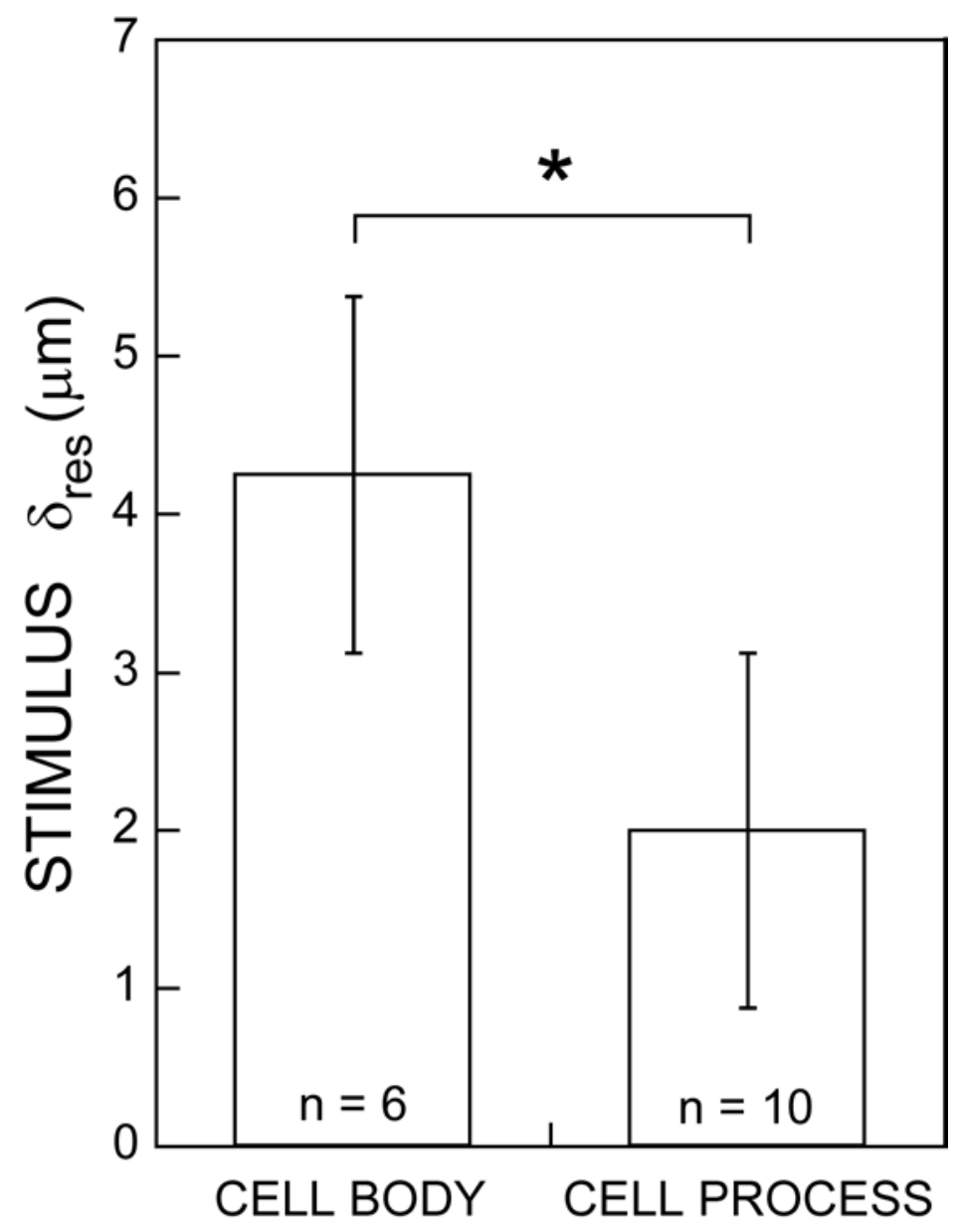




\section{Figure 6}

A

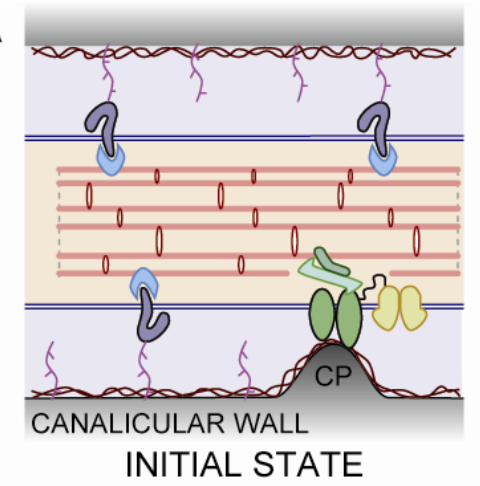

B

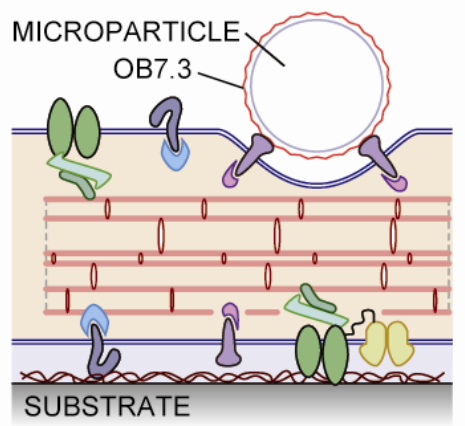

INITIAL STATE
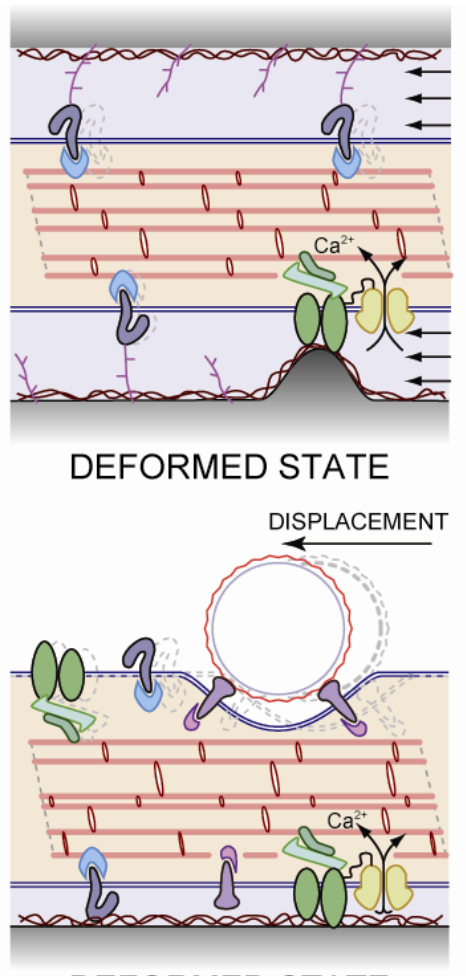

DEFORMED STATE
INTERSTITIAL FLUID FLOW

ACTIN FILAMENT

CELL MEMBRANE

CROSS-LINKING PROTEIN

TETHERING ELEMENT

ADHESION MOLECULE \& MEDIATED MOLECULE

CANALICULAR PROJECTION

EXTRACELLULAR MATRIX

INTEGRIN \&

MEDIATED MOLECULES

OB7.3

PHEX \& UNKNOWN MEDIATED MOLECULE

MECHANOSENSITIVE CHANNEL UNKNOWN CONNECTION

C

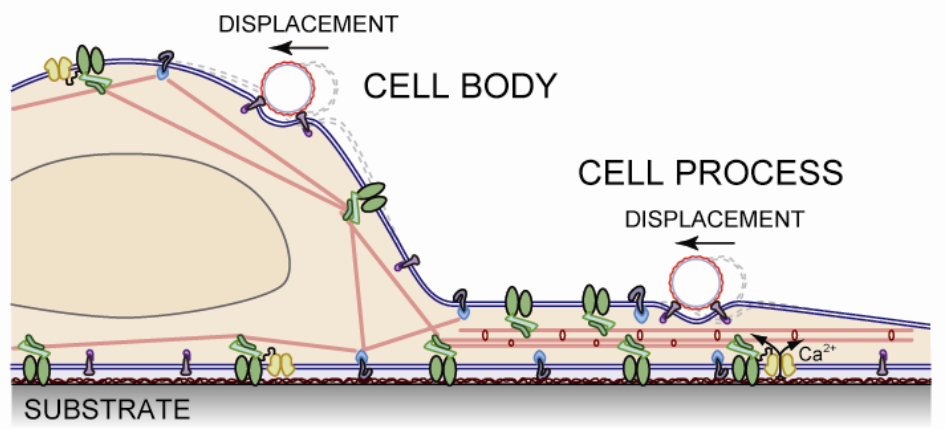

